\title{
Analysis of the Optimal Frequency Band for a Ballistic Missile Defense Radar System
}

\author{
Dang-An Nguyen ${ }^{1} \cdot$ Byoungho Cho $^{1} \cdot$ Chulhun Seo ${ }^{1, *} \cdot$ Jeongho Park $^{2} \cdot$ Dong-Hui Lee ${ }^{2}$
}

\begin{abstract}
In this paper, we consider the anti-attack procedure of a ballistic missile defense system (BMDS) at different operating frequencies at its phased-array radar station. The interception performance is measured in terms of lateral divert (LD), which denotes the minimum acceleration amount available in an interceptor to compensate for prediction error for a successful intercept. Dependence of the frequency on estimation accuracy that leads directly to prediction error is taken into account, in terms of angular measurement noises. The estimation extraction is performed by means of an extended Kalman filter (EKF), considering two typical re-entry trajectories of a non-maneuvering ballistic missile (BM). The simulation results show better performance at higher frequency for both tracking and intercepting aspects.
\end{abstract}

Key Words: Intercepting Prediction, Kalman Filter, Midway Guidance, Terminal Guidance, Tracking Radar.

\section{INTRODUCTION}

Ballistic missile defense systems (BMDSs) were developed to protect territories from enemy ballistic missiles (BMs) in the 1950s. Deployment of a BMDS allows a state to intercept any attacking missiles before they reach their intended targets [1]. It is possible to guide multiple interceptors by dedicated radars, stationary or otherwise, located on the ground, and to intercept BMs at different altitudes. The radar can begin tracking after the target has been launched and then communicate with controllers and launchers to release the interceptors and guide them, based on specific guidance settings [2,3]. It is naturally desirable for BMDSs to be able to intercept enemy BMs as rapidly as possible. This requires estimation accuracy during the tracking process, which is directly affected by the operating frequency at the radar station [4].

Frequency selection is difficult for a variety of reasons. Low frequency may be capable of covering long-range targets because the signal travels a longer distance, but the radar ranging resolution is large, owing to limited transmission bandwidth. Also, the dimension of phased-array radar increases significantly because of the large distance from antennae elements. High frequency provides more scalable bandwidth resources and optimal performance at short range, thanks to its small beamwidth [5]. Its drawbacks appear in the case of long-range targets, on account of free-space or atmospheric losses. The cost of radio frequency components also influences the frequency selection: the higher the frequency, the more expensive are the components. Therefore, no frequency can satisfy all conditions, and the radar only operates at the frequency that is subject to the least number of constraints. An appropriate frequency must be selected to provide optimal interception performance.

The purpose of the present study is to compare the interception performance at various frequencies for a non-maneuvering

Manuscript received February 23, 2018 ; Accepted May 5, 2018 ; Accepted June 19, 2018. (ID No. 20180223-024J)

${ }^{1}$ Department of Information Communication, Materials, and Chemistry Convergence Technology, Soongsil University, Seoul, Korea.

${ }^{2}$ LIG Nex1 Company, Seongnam, Korea.

*Corresponding Author: Chulhun Seo (e-mail: chulhun@ssu.ac.kr)

This is an Open-Access article distributed under the terms of the Creative Commons Attribution Non-Commercial License (http://creativecommons.org/licenses/by-nc/4.0) which permits unrestricted non-commercial use, distribution, and reproduction in any medium, provided the original work is properly cited.

(c) Copyright The Korean Institute of Electromagnetic Engineering and Science. All Rights Reserved. 
re-entry enemy BM. An allowable estimation error throughout the tracking process is determined, based on the acceleration capability of an interceptor to ensure successful destruction at the intended altitude. The non-linear motion and measurement models are discussed mathematically; these reflect the challenge of tracking precisely within the terminal phase of the BM. The extended Kalman filter (EKF) is chosen for estimation extraction, based on two typical trajectories in 3D Cartesian coordinate systems. The effectiveness of the EKF is measured by means of root-sum-squared position error, denoting the deviation between actual and estimated BM locations. The effect of frequency on the position error is discussed in terms of radar measurement noise, which results in a reduction in performance. The accuracy of estimations leads to precision in predicting the intercepting point where BM termination will happen. The lateral divert, known as the least amount of acceleration that an interceptor must attain for a successful intercept, is investigated from the viewpoint of a zero-lag terminal guidance system.

The arrangement of our material is as follows. Section II provides an overview of the basic functional principles of a fundamental BMDS. In Section III, problems and techniques used for the tracking process are thoroughly investigated in subsections on various coordinate systems, motion and measurement models, and EKF. Section IV discusses the basic terminal guidance system and related issues. The simulation results are shown in Section V. Conclusions are presented in Section VI.

\section{BALLISTIC MisSILE DEFENSE SYSTEM}

The BMDS is commonly a composite system of various components with different functions. The intercepting procedure of a BMDS is illustrated simply in Fig. 1.

In general, a BMDS is equipped with a ground radar station whose antenna can be a dipole, parabolic or phased-array, a command and control system, and a missile launcher, which can be integrated or located separately.

For an attacking-defense process, the radar starts tracking the BM, beginning from point $\mathrm{A}$, to obtain useful estimations of its position and velocity, then predicts an intercepting point $\mathrm{C}$, where the BM will be terminated. The predicted intercepting point can be calculated approximately based on the motion model of the target [3]; this information is sent to the command and control section. During the BM's flight, knowledge about the potential intercepting point continues to be updated and improved, and the interceptor is guided based on the midway guidance law until tracking at radar ends (assumed to be at B). When the interceptor is close enough or acquires the target (assumed to be at D), the seeker with which the missile is equipped operates as an active radar and takes over tracking through a terminal guidance system, before destroying the target in an

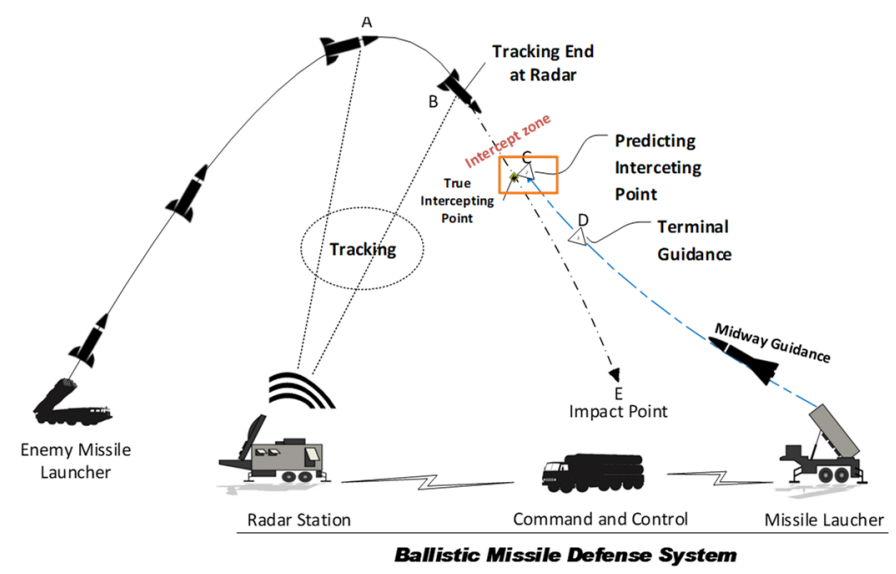

Fig. 1. Fundamental ballistic missile defense system.

allowable intercept zone, either by explosion (near-fuze) or collision (hit-to-kill). In Fig. 1, it is assumed that, when the seeker acquisition happens, the radar stops tracking. The entire defense procedure can be summarized in three main actions as:

- The radar tracks the target and predicts the location of the intercepting point.

- The prediction point is updated and the interceptor is guided under the midway guidance law.

- Terminal guidance happens when the interceptor is close enough to the target and radar tracking stops.

The phased-array radar is located on the ground and records the BM on its trajectory. The radar provides indirect measurements of the target, such as range and angle, i.e., azimuth and elevation, which are corrupted by radar noise, and useful estimation extraction is performed by a noise filter. The accuracy of the estimation depends greatly on the way that the radar operates, and can lead to a prediction error, for which the interceptor must compensate for a successful intercept. The precision of the predicted intercepting point directly influences interception performance. For example, if the missile cannot see the target owing to a large prediction error, this will lead to interception failure. Also, a failed hit will occur if the interceptor, though able to approach the target, does not have enough energy or acceleration available to correct the prediction error.

The operating frequency is one of the crucial factors impacting on prediction accuracy and effective operation of the radar station. Some specific radar frequency bands used for typical BMDSs can be found in [6].

There is no specific standard for choosing the frequency for the optimal design of the BMDS. However, it is possible to select an operating frequency from the point of view of performance.

\section{PHASED-ARRAY RADAR FOR TRACKING}

In this section, we consider how the radar works to obtain es- 
timations for the BMs, including the following issues: Cartesian coordinate systems, re-entry motion model, frequency-dependent radar measurement model, and EKF.

\section{Coordinate Systems}

For tracking purposes, the radar estimates the position and velocity of the target on Cartesian coordinate systems (CSs). Various CSs are commonly used, including earth-centered inertial (ECI) CS, earth-centered fixed (ECF, ECEF, or ECR) CS, east-north-up (ENU) CS, and radar face (RF) CS. More details on the first two CSs can be found in [7]. In the present work, we have selected ENU and RF CSs to express information on the target, as illustrated in Fig. 2.

The origin of the ENU CS (Oxyz) is located at the radar station above the reference Earth surface $h_{r}$ and its vertical axis $O z$ is directed along the local vertical line; $O x$ and $O y$ axes lie on the local horizontal plane, with $O x$ pointing east and $O y$ pointing north. The RF CS $O x^{\prime} y^{\prime} z^{\prime}$ is normally used in phased-array radar systems, rather than the ENU CS. Its origin is located at the radar face and the $O z^{\prime}$ axis is normal to the radar face; $O x^{\prime}$ and $O y^{\prime}$ axes lie on the radar face, with $O x^{\prime}$ lying along the intersection of the radar face and the local horizontal plane.

The radar face is fixed, and therefore the ENU and RF CSs can be transformed into each other through a transformation matrix $\mathbf{T}$ based on known deviation angles, as follows:

$$
\left[\begin{array}{l}
x \\
y \\
z
\end{array}\right]=\mathbf{T}\left[\begin{array}{l}
x^{\prime} \\
y^{\prime} \\
z^{\prime}
\end{array}\right]
$$

where

$$
\mathbf{T}=\left[\begin{array}{ccc}
\cos \lambda & \cos \phi \sin \lambda & \sin \phi \sin \lambda \\
-\sin \lambda & \cos \phi \cos \lambda & \sin \phi \cos \lambda \\
0 & -\sin \phi & \cos \phi
\end{array}\right]
$$

Note that $\mathbf{T}^{-1}=\mathbf{T}^{T}$ because the transformation matrix $\mathbf{T}$

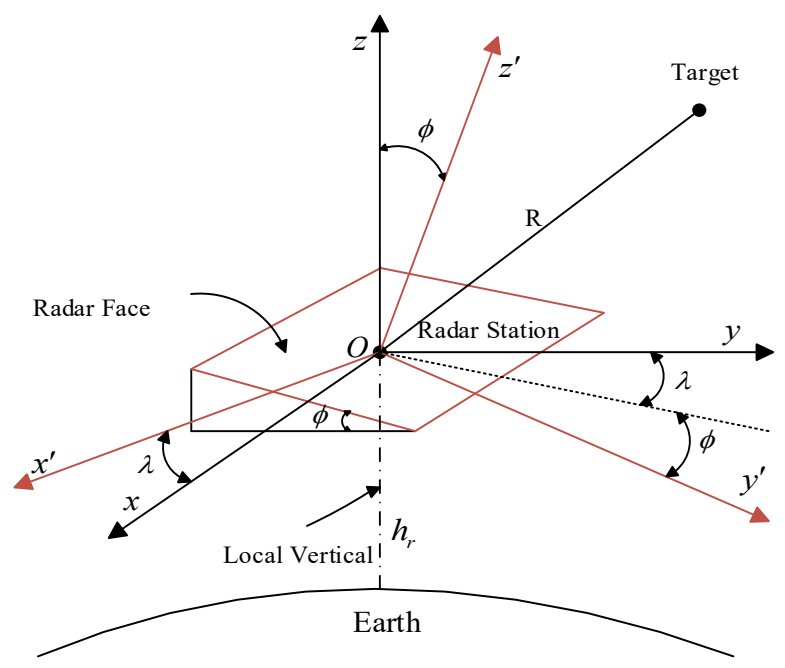

Fig. 2. East-north-up and radar-face coordinate systems. is orthogonal and the two Cartesian CSs coincide as $\phi=\lambda=$ 0 . This can reduce computational complexity, because both measurements and estimations of the target are expressed on the same CS.

\section{Re-entry Motion Model of BM}

In an entire trajectory of a BM, several different forces act on the missile, and not all trajectory regimes are influenced by the same number of forces. Therefore, it is difficult to portray the BM's full motion by employing a single model only. In many contexts, the BM's flight is commonly partitioned into three phases, as shown in Fig. 3.

- Boost: The BM is exposed to forces of thrust, drag, and gravity, and this phase lasts from the launch to the burnout, i.e., turn-off thrusters, around 4 minutes. The BM is powered and accelerated within endo-atmospheric flight.

- Midcourse: During an exo-atmospheric, free-flight motion, which lasts approximately 20 minutes, only gravity impacts on the BM.

- Re-entry: The BM re-enters the atmosphere, and the atmospheric drag becomes considerable, enduring until reaching the intended impact point. The drag-induced acceleration depends on the velocity and altitude of the BM [7].

It is possible and easier to conceive a more precise motion model of the BM within a particular phase. Because the earth model can be considered as flat, spherical, or ellipsoidal, the BM's motion is described in different forms, with a trade-off between complexity and accuracy. The relevant model is chosen for an optimal design, according to the point of view of the designer.

In the present work, we look at the re-entry phase only, drawing on the spherical earth model. As mentioned above, there are two main impacts on the BM during the re-entry phase, i.e., gravity and drag; however, in a maneuvering BM, lift force may be exerted on it, leading to more complicated estimating process.

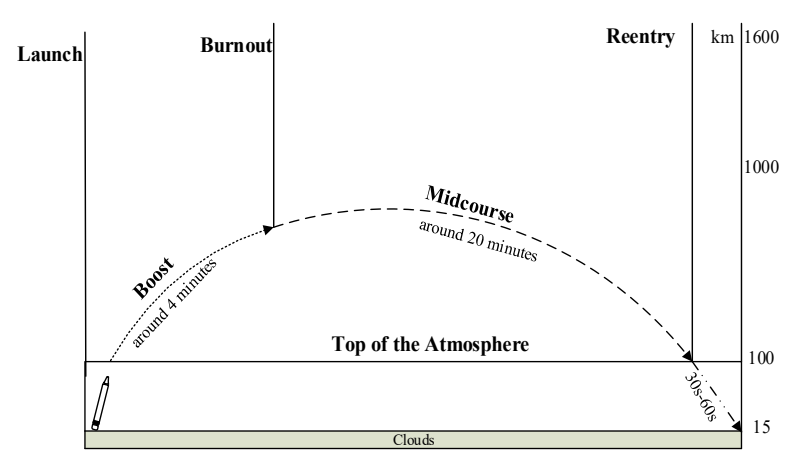

Fig. 3. Different trajectory phases of a BM. 
A non-maneuvering $\mathrm{BM}$ is our focus of interest. Also, depending on the CS used, a re-entry non-maneuvering BM traveling in the endo-atmosphere is not only subject to atmospheric drag and the Earth's gravity but also to the Coriolis and centrifugal forces [7].

A motion model of a $\mathrm{BM}$ is formed and expressed in the ENU Cartesian CS and with the assumption that the relevant information on the target includes the position and velocity. For the sake of convenience, we assume that $\mathbf{p}=[x, y, z]^{T}$ and $\mathbf{v}=\left[v_{x}, v_{y}, v_{z}\right]^{T}$ are two vectors denoting the position and the velocity of the target, respectively. The dynamic model at the reentry regime is usually described in a differential form as:

$$
\begin{gathered}
\dot{\mathbf{p}}=\mathbf{v} \\
\dot{\mathbf{v}}=\mathbf{a}_{d}+\mathbf{a}_{g}+\mathbf{a}_{\text {Coriolis }}+\mathbf{a}_{\text {centrifugal }}
\end{gathered}
$$

where $\mathbf{a}$ is total acceleration; $\mathbf{a}_{g}, \mathbf{a}_{d}, \mathbf{a}_{\text {Coriolis }}, \mathbf{a}_{\text {centrifugal }}$ are acceleration vectors induced by the Earth's gravity, the drag, the Coriolis, and the centrifugal force, respectively. Rewriting (3), we have:

$$
\dot{\mathbf{x}}=\left[\begin{array}{c}
\dot{\mathbf{p}} \\
\dot{\mathbf{v}}
\end{array}\right]=\left[\begin{array}{l}
\mathbf{v} \\
\mathbf{a}
\end{array}\right]
$$

where $\mathbf{x}=\left[x, y, z, v_{x}, v_{y}, v_{z}\right]^{T}$ represents the state vector of the target.

The total acceleration for the re-entry BM in the ENU CS is given specifically in [8], and is as follows:

$$
\begin{aligned}
{\left[\begin{array}{c}
\ddot{x} \\
\ddot{y} \\
\ddot{z}
\end{array}\right]=} & {\left[\begin{array}{c}
-2 \omega_{e}(\dot{z} \cos \varphi-\dot{y} \sin \varphi) \\
-\omega_{e}^{2} \sin \varphi\left[\left(r_{0}+z\right) \cos \varphi-y \sin \varphi\right] \\
\omega_{e}^{2} \cos \varphi\left[\left(r_{0}+z\right) \cos \varphi-y \sin \varphi\right.
\end{array}\right] } \\
& +\left[\begin{array}{c}
\omega_{e}^{2} x-\frac{\rho \mathrm{g}_{0} V \dot{x}}{2 \beta}+\frac{\mathrm{g} x}{r_{1}} \\
-2 \omega_{e}(\dot{x} \sin \varphi)-\frac{\rho \mathrm{g}_{0} V \dot{y}}{2 \beta}+\frac{\mathrm{g} y}{r_{1}} \\
2 \omega_{e}(\dot{x} \cos \varphi)-\frac{\rho \mathrm{g}_{0} V \dot{z}}{2 \beta}+\frac{\mathrm{g}\left(z+r_{0}\right)}{r_{1}}
\end{array}\right]
\end{aligned}
$$

where

$$
\begin{gathered}
V=\sqrt{\dot{x}^{2}+\dot{y}^{2}+\dot{z}^{2}}=\text { missile velocity }(\mathrm{ft} / \mathrm{s}) \\
\beta=\text { ballistic coefficient } \\
\mathrm{g}=\mathrm{g}_{0} r_{0}^{2} / r_{1}^{2}
\end{gathered}
$$

$\mathrm{g}_{0}=$ gravitational acceleration at sea level $\left(\mathrm{ft} / \mathrm{s}^{2}\right)$

$$
\begin{gathered}
\varphi=\text { latitude of the radar station } \\
r_{0}=\text { Earth's radius }(\mathrm{ft}) \\
r_{1}=\sqrt{x^{2}+y^{2}+\left(z+r_{0}\right)^{2}}
\end{gathered}
$$

$=$ distance from the center of the Earth to the missile $(\mathrm{ft})$

$$
\begin{gathered}
\omega_{e}=\text { rotation rate of the Earth }(\mathrm{rad} / \mathrm{s}) \\
\quad \rho=\rho_{0} e^{-K h}=\text { air density } \\
\\
\rho_{0}, K=\text { known parameters } \\
h=r_{1}-r_{0}=\text { altitude of the missile. }
\end{gathered}
$$

For the spherical model, the air density is an exponential function of altitude. The ballistic coefficient is known as the inverse drag parameter, given by $\alpha=S c_{D} / m$, where $m$ denotes target mass, $S$ denotes reference area, and $c_{D}$ is drag coefficient. The drag parameter is unknown and not constant; therefore, in the present work, an unknown drag-related parameter $\rho / \beta$ is added to the state vector and estimated online to enhance performance.

Ultimately, the complete state vector of the BM is $\mathbf{x}=$ $[x, y, z, \dot{x}, \dot{y}, \dot{z}, \rho / \beta]^{T}$. Let $x_{1}=x, x_{2}=y, x_{3}=z, x_{4}=\dot{x}$, $x_{5}=\dot{y}, x_{6}=\dot{z}, x_{7}=\rho / \beta$. The dynamic motion model is given by [9], and is as follows:

$$
\begin{aligned}
& \dot{\mathbf{x}}=\left[\begin{array}{c}
\dot{x} \\
\dot{y} \\
\dot{z} \\
\ddot{x} \\
\ddot{y} \\
\ddot{z} \\
\dot{\rho} \\
\bar{\beta}
\end{array}\right] {\left[\begin{array}{c}
x_{4} \\
x_{5} \\
x_{6} \\
-\omega_{e}^{2} \sin \varphi\left[\left(r_{0}+x_{3}\right) \cos \varphi-x_{2} \sin \varphi\right] \\
\omega_{e}^{2} \cos \varphi\left[\left(r_{0}+x_{3}\right) \cos \varphi-x_{2} \sin \varphi\right. \\
-K x_{7}\left[x_{1} x_{4}+x_{2} x_{5}+\frac{\left(x_{3}+r_{0}\right) x_{6}}{r_{1}}\right]
\end{array}\right] } \\
& 0 \\
& 0 \\
& 0 \\
&+\left[\begin{array}{c}
\omega_{7}^{2} x_{1}-\frac{x_{7} V x_{4}}{2}+\frac{\mathrm{g} x_{1}}{r_{1}} \\
-2 \omega_{e}\left(x_{4} \sin \varphi\right)-\frac{x_{7} \mathrm{~g}_{0} V x_{5}}{2}+\frac{\mathrm{g} x_{2}}{r_{1}} \\
2 \omega_{e}\left(x_{4} \cos \varphi\right)-\frac{x_{7} \mathrm{~g}_{0} V x_{6}}{2}+\frac{\mathrm{g}\left(x_{3}+r_{0}\right)}{r_{1}} \\
0
\end{array}\right]
\end{aligned}
$$

(6) is the non-linear function of state vector $\mathbf{x}$.

$$
\dot{\mathbf{x}}=\mathbf{f}(\mathbf{x})
$$

where $\mathbf{f}(\mathbf{x})$ is the seven-dimensional vector function of $\mathbf{x}$. The state vector of the target can be discretized by expanding $\mathbf{x}=$ $\mathbf{x}(t+\Delta t)$ by Taylor expansion up to the first order:

$$
\mathbf{x}(t+\Delta t)=\mathbf{x}(t)+\dot{\mathbf{x}}(t) \Delta t+\text { HOT }
$$

where $\Delta t$ denotes the small-time step and HOT denotes higher order terms. Defining $\mathbf{x}_{k}=\mathbf{x}(t)$ and $\mathbf{x}_{k+1}=\mathbf{x}(t+\Delta t)$, (8) can be rewritten as follows:

$$
\mathbf{x}_{k+1}=\mathbf{x}_{k}+\mathbf{f}\left(\mathbf{x}_{k}\right) \Delta \mathrm{t}+\mathbf{q}_{k}
$$

where $\mathbf{q}_{k}$ represents the discretization error (including HOT) and modeling uncertainties in motion, and (9) is the recursive motion equation of the re-entry BM. It is assumed that the error $\mathbf{q}_{k}$ is Gaussian, zero-mean, and white:

$$
E\left\{\mathbf{q}_{k}\right\}=0, E\left\{\mathbf{q}_{k} \mathbf{q}_{k}^{T}\right\}=\mathbf{Q}_{k} \boldsymbol{\delta}_{k j}
$$


where $\delta_{k j}=1$ for $k=j$ and $\delta_{k j}=0$ for others. Note that $\mathbf{Q}_{k}$ is a covariance matrix and is one of the known parameters for the filtering technique discussed in later sections.

\section{Radar Measurement Model}

In this section, we present a measurement model for phasedarray radar. As is known, phased-array radar measures the range and angular information of the BM on a spherical CS, which is referenced directly to the RF Cartesian CS $\left(O x^{\prime} y^{\prime} z^{\prime}\right)$.

Specifically, the phased-array radar used for tracking provides range $r$, which denotes distance between the radar and the target, and two angular measurements, i.e., azimuth $b$ and elevation $e$, as illustrated in Fig. 4.

In the spherical CS, these measurements are generally modeled in the following form of additive noise:

$$
\begin{aligned}
& r=\mathrm{r}+w_{r} \\
& b=\mathrm{b}+w_{b} \\
& e=\mathrm{e}+w_{e}
\end{aligned}
$$

where $r, b$, and $e$, which are in non-italic form, denote true measurements of the target in the sensor spherical CS, and $w_{r}, w_{b}$, and $w_{e}$ represent the uncorrelated Gaussian noises with zero-mean, as:

$$
E\{\mathbf{w}\}=0, \mathbf{R}=E\left\{\mathbf{w} \mathbf{w}^{T}\right\}=\operatorname{diag}\left(\sigma_{r}^{2}, \sigma_{b}^{2}, \sigma_{e}^{2}\right)
$$

where $\mathbf{w}=\left[w_{r}, w_{b}, w_{e}\right]^{T}$ is the measurement noise vector and $\mathbf{R}$ denotes the covariance matrix, which is the known parameter. Some other measurement models can be found in [10].

Let $\left(x^{\prime}, y^{\prime}, z^{\prime}\right)$ be the true position of the BM on the RF Cartesian CS. The noise-corrupted measurements can be converted into Cartesian coordinates as:

$$
\left[\begin{array}{l}
r \\
b \\
e
\end{array}\right]=\left[\begin{array}{c}
\sqrt{x^{\prime 2}+y^{\prime 2}+z^{\prime 2}} \\
\tan ^{-1}\left(y^{\prime} / x^{\prime}\right) \\
\tan ^{-1}\left(z^{\prime} / \sqrt{x^{\prime 2}+y^{\prime 2}}\right)
\end{array}\right]=\mathbf{g}(\mathbf{x})
$$

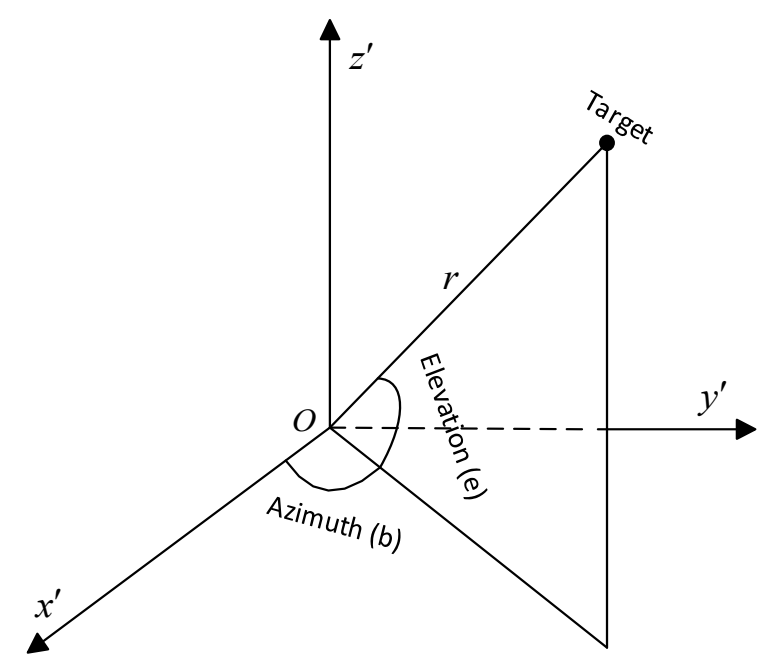

Fig. 4. Radar measurement model.
Clearly, the measurements relate to the state vector $\mathbf{x}$ in a non-linear function $\mathbf{g}$, and, after adding a time index (11), become:

$$
\mathbf{y}_{k}=\mathbf{g}\left(\mathbf{x}_{k}\right)+\mathbf{w}_{k}
$$

The measurement equation is given by (14), where $\mathbf{y}_{k}=$ $[r, b, e]_{k}^{T}$ and $\mathbf{w}_{k}=\left[w_{r}, w_{b}, w_{e}\right]_{k}^{T}$ denote the noise- corrupted measurement vector and the radar noise vector, respectively, at time $k$, and $\mathbf{g}$ is the vector function of $\mathbf{x}_{k}$. The measurement noise comes from several different sources, and it is impossible to devise a perfect system without noise. The dependence of noise on frequency is one of the factors needing to be clarified.

In general, accuracy of each measurement is represented by standard deviation $\sigma$. According to [5], there are three main noise sources causing range measurement error that is modelled by range standard deviation $\sigma_{r}$ as:

$$
\sigma_{r}=\sqrt{\sigma_{R N}^{2}+\sigma_{R F}^{2}+\sigma_{R B}^{2}}
$$

where

$$
\begin{aligned}
& \sigma_{R N}=\text { SNR-dependent random range error, } \\
& \sigma_{R F}=\text { range fixed random error, } \\
& \sigma_{R B}=\text { range bias error. }
\end{aligned}
$$

The SNR-dependent random range measurement error dominates the radar range error and is determined as follows:

$$
\sigma_{R N}=\Delta R / \sqrt{2(\mathrm{SNR})}
$$

where $\Delta R=c \tau / 2$ is the range resolution, $\tau$ the pulse-width, $c$ the light speed; and signal-to-noise ratio (SNR) is known as the radar sensitivity.

Similarly, accuracy of two angular measurements (azimuth and elevation angles) is also determined by the root-sumsquared standard deviation of three main errors, as:

$$
\sigma_{A}=\sqrt{\sigma_{A N}^{2}+\sigma_{A F}^{2}+\sigma_{A B}^{2}}
$$

where

$$
\begin{aligned}
& \sigma_{A N}=\text { SNR-dependent random range error, } \\
& \sigma_{A F}=\text { range fixed random error, } \\
& \sigma_{A B}=\text { range bias error. }
\end{aligned}
$$

The SNR-dependent random angular measurement error dominates the radar angular error and is given by:

$$
\sigma_{A N}=\theta / k_{M} \sqrt{2(\mathrm{SNR})}
$$

where $k_{M}$ is the mono-pulse pattern difference slope and typically equal to 1.6 , and $\theta$ is the half-power broadside beamwidth in the angular coordinate of the measurement. The accuracy of the azimuth and elevation angles is identified corresponding to the beamwidth values on the respective plane. For 
phased-array radar, the broadside beamwidth on each angular coordinate can be broadened by a scan angle off-broadside $\gamma$, leading to the angular error increment $\sigma_{A N}$, as:

$$
\sigma_{A N}=\theta / k_{M} \cos \gamma \sqrt{2(\mathrm{SNR})}
$$

The broadside beamwidth relates to wavelength or operating frequency and antenna size, as follows:

$$
\theta=k_{A} c / f D
$$

where $k_{A}$ is the antenna beamwidth coefficient and nearly unity, $f$ is the operating frequency, and $D$ is the dimension of the antenna on the plane at which antenna patterns are measured. For example, a rectangular phased-array antenna of size $L \times W$ is capable of steering the beam in two dimensions; the broadside beamwidths on azimuth and elevation coordinates can be calculated as:

$$
\begin{aligned}
\theta^{\text {azimuth }} & ={ }^{k_{A} c} / f L \\
\theta^{\text {elevation }} & =k_{A} c / f W
\end{aligned}
$$

\section{Extended Kalman Filter}

The Kalman filter $(\mathrm{KF})$ is a highly adaptable iterative algorithm, which can estimate non-measured quantities [11]. In radar applications, the BM velocity is not provided directly by radar measurements; therefore, $\mathrm{KF}$ is a useful tool for extracting the entire state of the target. The motion and measurement equations are known as the recognized knowledge of the $\mathrm{KF}$, and are constructed in linear forms. The EKF is broadened to apply to non-linear systems [12]. We restate the motion and measurement equation of $\mathrm{BM}$ mentioned above as:

$$
\begin{aligned}
\mathbf{x}_{k+1} & =\mathbf{x}_{k}+\mathbf{f}\left(\mathbf{x}_{k}\right)+\mathbf{q}_{k} \\
\mathbf{y}_{k} & =\mathbf{g}\left(\mathbf{x}_{k}\right)+\mathbf{w}_{k}
\end{aligned}
$$

For the sake of convenience, let $\hat{\mathbf{x}}_{k / j}$ denote the estimate of $\mathbf{x}_{k}$ based on measurements up to time $j$, and $\mathbf{P}_{k / j}$ denote the error covariance matrix associated with $\hat{\mathbf{x}}_{k / j}$. The whole procedure of EKF can be concisely summarized in the following equations:

- State prediction equation

$$
\hat{\mathbf{x}}_{k+1 / k}=\hat{\mathbf{x}}_{k / k}+\mathbf{f}\left(\hat{\mathbf{x}}_{k / k}\right) \Delta t
$$

- State correction equation

$$
\hat{\mathbf{x}}_{k+1 / k}=\hat{\mathbf{x}}_{k+1 / k}+\mathbf{K}_{k+1}\left(\mathbf{y}_{k+1}-\mathbf{g}\left(\hat{\mathbf{x}}_{k+1 / k}\right)\right)
$$

where $\mathbf{K}_{k+1}$ denotes the filter gain.

$$
\mathbf{K}_{k+1}=\mathbf{P}_{k+1 / k} \mathbf{G}_{k+1}^{T}\left(\mathbf{G}_{k+1} \mathbf{P}_{k+1 / k} \mathbf{G}_{k+1}^{T}\right)
$$

- Covariance prediction equation

$$
\begin{gathered}
\mathbf{P}_{k+1 / k}=\mathbf{F}_{k} \mathbf{P}_{k / k} \mathbf{F}_{k}^{T}+\mathbf{Q}_{k} \\
\mathbf{F}_{k}=\mathbf{I}+\mathbf{A}\left(\hat{\mathbf{x}}_{k / k}\right) \Delta t
\end{gathered}
$$

where $\mathbf{A}$ is a Jacobian matrix of function $\mathbf{f}$ and is defined as:

$$
\mathbf{A}\left(\hat{\mathbf{x}}_{k / k}\right)=\partial \mathbf{f} /\left.\partial \mathbf{x}\right|_{\hat{\mathbf{x}}_{k / k}}
$$

each element being calculated as in [9].

- Covariance correction equation

$$
\mathbf{P}_{k+1 / k+1}=\left(\mathbf{I}-\mathbf{K}_{k+1} \mathbf{G}_{k+1}\right) \mathbf{P}_{k+1 / k}
$$

where $\mathbf{G}$ is a Jacobian matrix of function $\mathbf{g}$, as:

$$
\mathbf{G}_{k+1}=\partial \mathbf{g} /\left.\partial \mathbf{x}\right|_{\hat{\mathbf{x}}_{k+1 / k}}
$$

each element being given in the Appendix.

The detailed flow diagram of EKF for the filtering problem can be found in [9].

\section{TERMINAL GUIDANCE SYSTEM}

Before launching an interceptor, the radar tracks the BM and predicts an intercepting point in advance. The interceptor is then guided by the midway guidance law to move to that intercepting point. During the flight of the intercepting missile, the location of the intercepting point continues to be updated until seeker acquisition happens, when the interceptor is close enough and can see the target. If the target's future location is known perfectly, a missile guidance system inside the interceptor is not necessary, because there are no errors to allow for. However, it is impossible to know the intercepting point precisely; therefore, the launching interceptor may be flown in the wrong direction, such an error being the main factor causing fail intercept.

Once the seeker sees the target, the terminal guidance activates, and the seeker plays a role as active radar, taking over the tracking throughout the remaining time until intercept. The intercepting missile supplies an acceleration amount whose direction is perpendicular to its velocity direction, by fuel burn or removing its control surface [3]. The commanded amount of acceleration depends on the heading error, and takes the form of the proportional navigation law, which is given as:

$$
n_{c}=N^{\prime} V_{c} \dot{\delta}
$$

where $n_{c}$ is the acceleration command (in $\mathrm{ft} / \mathrm{s}^{2}$ ), $N^{\prime}$ is a unitless, designer-chosen gain, known as the effective navigation ratio, and is usually within a range as set out in $[3,5] ; V_{c}$ is the missile-target closing velocity (in $\mathrm{ft} / \mathrm{s}$ ), and the line of sight angle $\delta$ (in rad) is the angle between an imaginary line connecting the interceptor and the ballistic target and a fixed reference, as illustrated in Fig. 5. The over-dot denotes the time derivative 


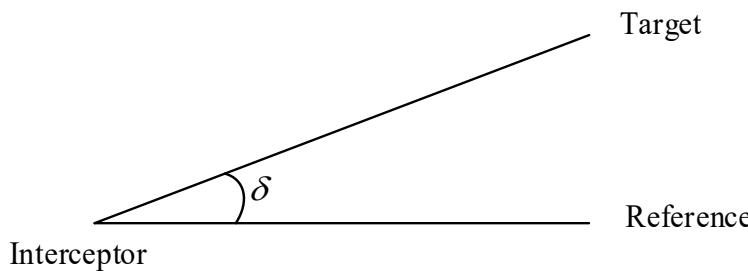

Fig. 5. Line of sight angle.

of the line of sight angle. More detailed information on the proportional navigation law can be found in [13].

A diagram of a typical terminal guidance system takes the form of a control loop, as shown in Fig. 6 [13]. In this diagram, the interceptor acceleration $n_{L}$ is subtracted from the target acceleration to generate a relative acceleration, and then a relative distance is formed after two integrations; at the end of the flight, the relative distance, called miss distance, is considered as a performance parameter. Most missile designers desire zeromiss distance. The line of sight angle $\delta$ is extracted by heading-error addition. For a zero-lag guidance system (not dynamic) and a non-maneuvering target, the miss distance will always be zero if the interceptor has sufficient acceleration to offset heading error throughout the seeker acquisition time.

If a zero-miss distance determines a successful intercept, the required acceleration to compensate for heading error at an instant time within flight time $t_{F}$ or the amount of time from seeker acquisition until intercept is given by:

$$
n_{c}=\left[-V_{M} H E N^{\prime}\left(1-t / t_{F}\right)^{N^{\prime}-2}\right] / t_{F}
$$

where $V_{M}$ is the velocity of the interceptor, $H E$ is the angular heading error, and $t$ is instantaneous time.

The prediction error $(\mathrm{PE})$ (in $\mathrm{ft}$ ) and the heading error have a relationship according to:

$$
\mathrm{PE}=-V_{M} H E t_{F}
$$

Substituting (35) into (34), we have

$$
n_{c}=\left[\operatorname{PEN} N^{\prime}\left(1-t / t_{F}\right)^{N^{\prime}-2}\right] / t_{F}^{2}
$$

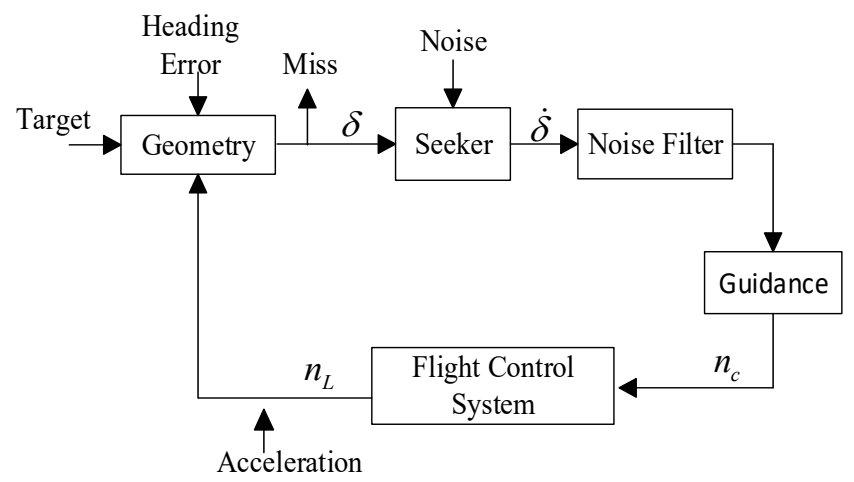

Fig. 6. Terminal guidance system.
The lateral divert or total acceleration $\Delta V$ required during the flight time $t_{F}$ relates to $n_{c}$ according to:

$$
\begin{aligned}
\Delta V & =\int_{0}^{t_{F}}\left|n_{c}\right| d t \\
& ={ }^{P E} N^{\prime} /\left(N^{\prime}-1\right) t_{F}
\end{aligned}
$$

(37) indicates the minimum amount of lateral divert that must be available in an interceptor to ensure successful destruction. It can be seen that the longer the flight time, the smaller the lateral divert; therefore, techniques increasing the seeker acquisition range increase the acceleration capability of the interceptor.

\section{SIMULATION RESULTS}

In simulation, we consider two typical trajectories of the BM in its re-entry phase [8]. The BM at each trajectory is assumed to have the same ballistic coefficient and begin its re-entry phase at different altitudes with (nearly) the same beginning velocity. The actual initial state $\mathbf{x}_{0}$ includes the following elements: $x_{1}=338,110 \mathrm{ft}, x_{2}=338,110 \mathrm{ft}, x_{3}=199,910 \mathrm{ft}, x_{4}=$ $-15,297 \mathrm{ft} / \mathrm{s}, x_{5}=-15,297 \mathrm{ft} / \mathrm{s}, x_{6}=-8,653 \mathrm{ft} / \mathrm{s}, x_{7}=4.395 \times$ $10^{-10} \mathrm{lb} / \mathrm{ft}^{2}$ for case 1 , and $x_{1}=920,640 \mathrm{ft}, x_{2}=451,515$ $\mathrm{ft}, \quad x_{3}=327,897 \mathrm{ft}, \quad x_{4}=-18,187 \mathrm{ft} / \mathrm{s}, \quad x_{5}=-11,232 \mathrm{ft} / \mathrm{s}$, $x_{6}=-7,014 \mathrm{ft} / \mathrm{s}, x_{7}=7.6742 \times 10^{-13} \mathrm{lb} / \mathrm{ft}^{2}$ for case 2 .

The effectiveness of EKF is compared across five frequency bands: L-band (1.3 GHz), S-band (2.5 GHz), C-band (5.5 $\mathrm{GHz}), \mathrm{X}$-band $(9 \mathrm{GHz})$, and $\mathrm{Ku}$-band $(13.5 \mathrm{GHz})$, by position error given by:

$$
\begin{aligned}
& \text { Position error }= \\
& \quad \sqrt{\left(x_{1}-\hat{x}_{1}\right)^{2}+\left(x_{2}-\hat{x}_{2}\right)^{2}+\left(x_{3}-\hat{x}_{3}\right)^{2}}
\end{aligned}
$$

The position error is averaged over Monte-Carlo simulation runs. At the radar, the sampling interval is set at $\Delta t=0.1 \mathrm{~s}$, the radar sensitivity $\mathrm{SNR}=12 \mathrm{~dB}$, and the pulse-width $\tau=1 \mu$ s. The phased-array rectangular antenna has a size of $3 \mathrm{~m} \times 5 \mathrm{~m}$, the scan angle off-broadside $\gamma=30^{\circ}$, the measurement covariance matrix $\mathbf{R}$ is given by (12), whose range variance is given by (15), and angular variances are calculated by (19).

Fig. 7 shows the actual altitude of the BM during re-entry flight time at (nearly) the same beginning velocity (around 23 $\mathrm{kft} / \mathrm{s})$. The BM at higher altitude takes a longer interval than lower-altitude BM to reach the same altitude. For example, the $\mathrm{BM}$ in case 2 flies to an altitude of $100 \mathrm{kft}$ in 40 seconds, and in just 12 seconds in case 1 . Also, the BM in case 1 is decelerated faster, owing to a higher drag effect at lower altitude, and vice versa, as shown in Fig. 8.

Figs. 9 and 10 show the position error during the tracking 


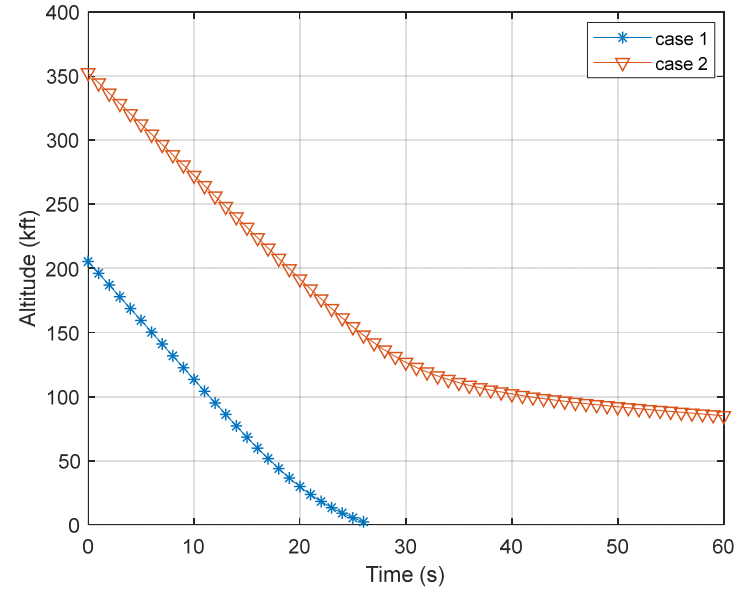

Fig. 7. Target's actual altitude during re-entry flight time.

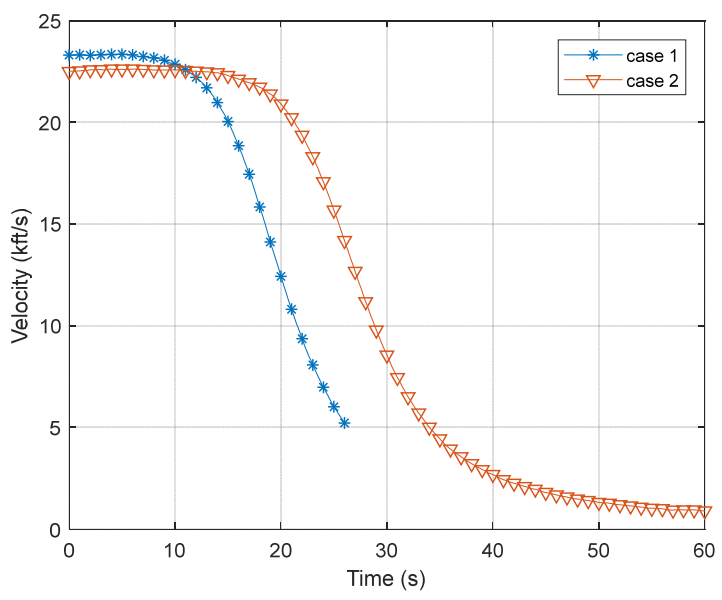

Fig. 8. Target's actual velocity versus re-entry flight time.

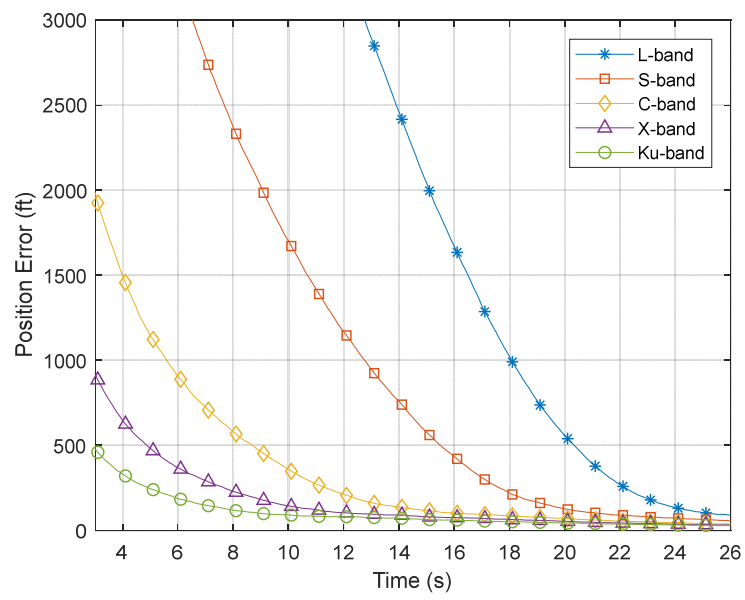

Fig. 9. The position error versus re-entry flight time for case 1: $\Delta \mathrm{t}$ $=0.1, \mathrm{SNR}=12 \mathrm{~dB}$.

time. It can be seen that the position error reduces as the time increases. The higher frequency yields a smaller position error. For example, the S-band radar derives a position error of about $3,000 \mathrm{ft}$, compared with just $800 \mathrm{ft}$ for the $\mathrm{C}$-band at the same tracking time of 15 seconds in case 1 . The performance gap is

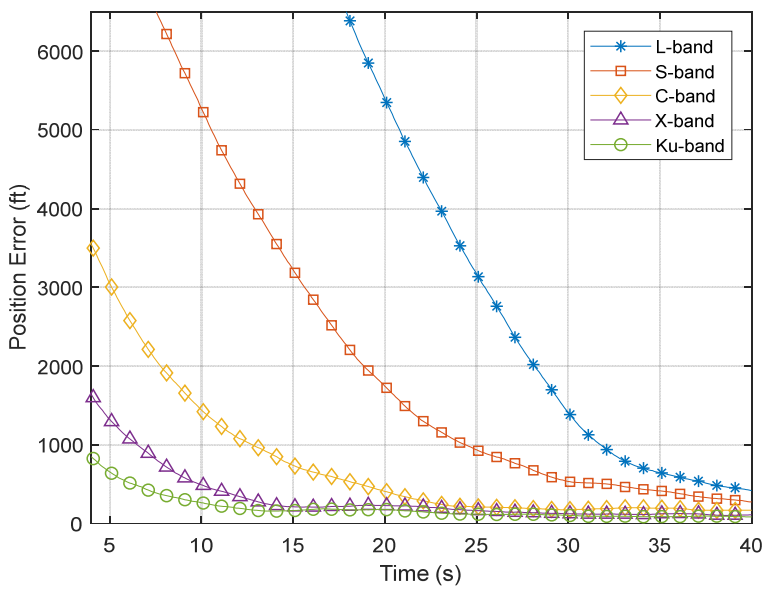

Fig. 10. The position error versus re-entry flight time for case 2: $\Delta \mathrm{t}$ $=0.1, \mathrm{SNR}=12 \mathrm{~dB}$.

negligible after 26 seconds and 40 seconds for case 1 and case 2 , respectively.

Figs. 11 and 12 show the lateral divert for an interceptor to correct the prediction error at an intercepting point of altitude $100 \mathrm{kft}$. According to Fig. 7, the time to reach the altitude of $100 \mathrm{kft}$ is 12 seconds for case 1 and 40 seconds for case 2. The flight time is assumed to be $t_{F}=3$ seconds for both trajectory cases. Note that the flight time is the time from the point at which tracking stops at radar until the intercepting time, meaning that the radar stops tracking at $t=9$ seconds and $t=37 \mathrm{sec}^{-}$ onds for case 1 and case 2 , respectively. It can be seen that the lateral divert reduces when the radar operates at a higher frequency, owing to smaller position error. For example, if a fixed capability of an interceptor is $600 \mathrm{ft} / \mathrm{s}$ for case 1 , the radar must operate at a frequency greater than $2.5 \mathrm{GHz}$ for a successful intercept. Also, the performance gap is negligible at a frequency larger than $5.5 \mathrm{GHz}$. When increasing the effective navigation ratio $N$, the missile needs less lateral divert; however, the guid-

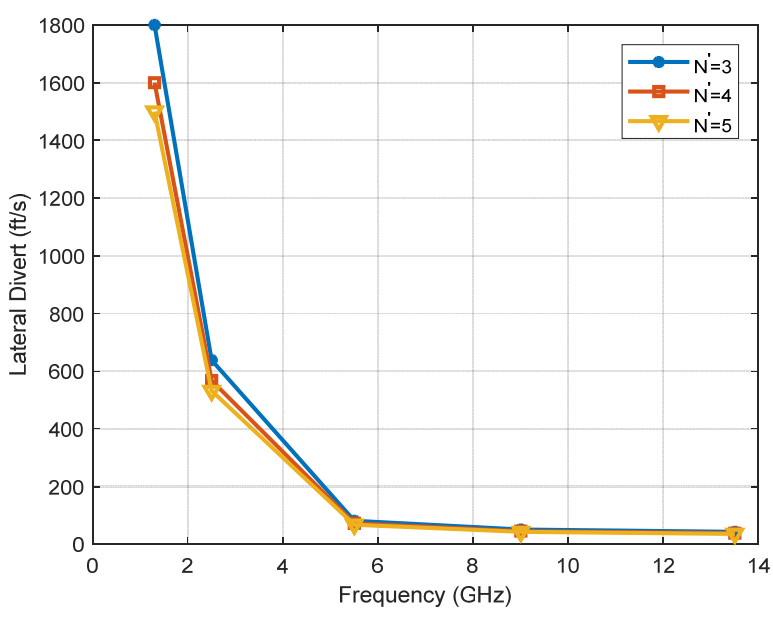

Fig. 11. Lateral divert versus frequency for zero-lag guidance system for case 1, with intercepting altitude of $100 \mathrm{kft}$ (about $30 \mathrm{~km}$ ), and flight time of $t_{F}=3$ seconds. 


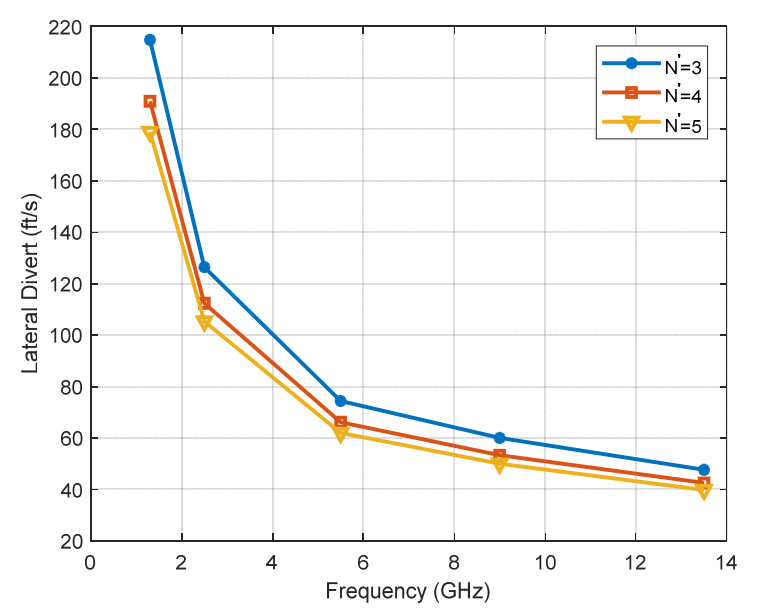

Fig. 12. Lateral divert versus frequency for zero-lag guidance system in case 2, with intercepting altitude of $100 \mathrm{kft}$ (about $30 \mathrm{~km}$ ), and flight time of $t_{F}=3$ seconds.

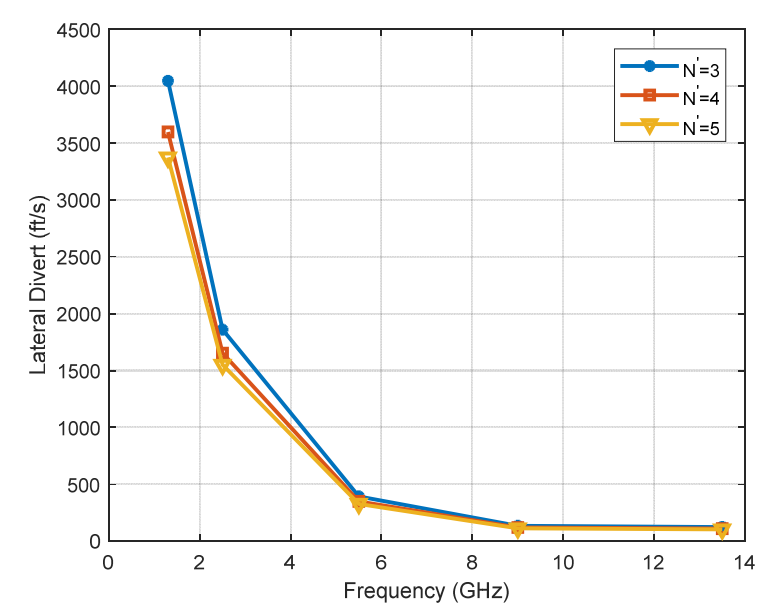

Fig. 13. Lateral divert versus frequency for zero-lag guidance system in case 2, with intercepting altitude of $230 \mathrm{kft}$ (about $70 \mathrm{~km}$ ), and flight time of $t_{F}=3$ seconds.

ance noise will increase significantly [14]. Furthermore, the lateral divert required in case 2 is much less than that in case 1 , since the altitude of the BM in case 2 is higher than in case 1. This causes the BM to travel for a longer time in order to reach the intercepting point, and the estimation is therefore improved.

This is also obvious when considering the higher altitude of the intercepting point shown in Fig. 13. The lateral divert in order to intercept at altitude $70(\mathrm{~km})$ is much larger than that at $30(\mathrm{~km})$. For example, at S-band, the interceptor must respond by an amount of more than 1,500 (ft/s) at an intercepting altitude of $70 \mathrm{~km}$, compared to around $120(\mathrm{ft} / \mathrm{s})$ at an intercepting altitude of $30(\mathrm{~km})$. The lateral divert gap between frequencies is also broadened.

This work was supported by the National Rsearch Foundation of Korea(NRF) grant fund by the Korean government(MSIP) (NRF-2017R1A5A 1015596).

\section{CONCLUSION}

In conclusion, the accuracy of the radar angular measurements is inverse to the frequency. The tracking performance is therefore improved at high frequency. This increases the intercepting capability of the BMDS, especially at high intercepting altitude.

\section{REFERENCES}

[1] A. Blencowe, "Pursuing peace with the weapons of war: ballistic missile defence and international security," 2009; https://www.e-ir.info/2009/09/05/pursuing-peace-with-theweapons-of-war-ballistic-missile-defence-and-internationalsecurity/.

[2] Y. Y. Chen and K. Y. Young, "An intelligent radar predictor for high-speed moving-target tracking," in Proceedings of 2002 IEEE Region 10 Conference on Computers, Communications, Control and Power Engineering, Beijing, China, 2002, pp. 1638-1641.

[3] P. Zarchan, "Ballistic missile defense guidance and control issues," Science E Global Security, vol. 8, no. 1, pp. 99-124, 1999.

[4] M. A. Richards, J. Scheer, and W. A. Holm, Principles of Modern Radar: Basic Principles. Raleigh, NC: SciTech Publishing, 2010.

[5] G. Richard Curry, Radar System Performance Modeling, 2nd ed. Boston, MA: Artech House, 2005.

[6] IEEE Standard for letter designations for radar-frequency bands (revision of IEEE 521-1984), IEEE 521-2002, 2002.

[7] X. R. Li and V. P. Jilkov, "Survey of maneuvering target tracking. Part II: Motion models of ballistic and space targets," IEEE Transactions on Aerospace and Electronic Systems, vol. 46, no. 1, pp. 96-119, 2010.

[8] Y. Kashiwagi, Prediction of Ballistic Missile Trajectories. Menlo Park, CA: Stanford Research Institute, 1968.

[9] M. Dressler and W. Ross, Real Time Implementation of the Kalman Filter for Trajectory Estimation. Menlo Park, CA: Stanford Research Institute, 1968.

[10] X. R. Li and V. P. Jilkov, "Survey of maneuvering target tracking. III. Measurement models," in Signal and Data Processing of Small Targets 2001. Bellingham, WA: International Society for Optics and Photonics, 2001, pp. 423-447.

[11] R. E. Kalman, "A new approach to linear filtering and prediction problems," Journal of Basic Engineering, vol. 82, no. 1, pp. 35-45, 1960.

[12] M. I. Ribeiro, "Kalman and extended Kalman filters: concept, derivation and properties," Institute for Systems and Robotics, Lisbon, Portugal, 2004.

[13] P. Zarchan, Tactical and Strategic Missile Guidance, 6th ed. 
Washington, DC: American Institute of Aeronautics and Astronautics Inc., 2012.

\section{Dang-An Nguyen}

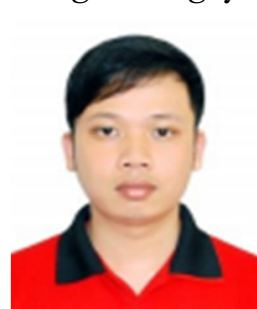

has a degree from the School of Electronics and Telecommunications at Hanoi University of Science and Technology, Vietnam. He has three years' experience working as a senior member of the Signal Processing and Radio Communication Laboratory. $\mathrm{He}$ is currently studying for a Master's degree at Soongsil University, Korea, where his major relates to microwave signal processing, radar systems, power amplifiers, and non-Foster circuits.

Byoungho Cho

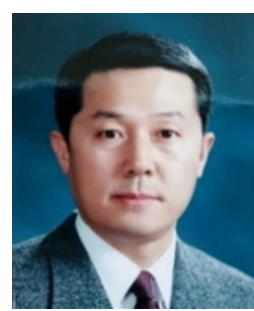

received his B.S. degree in electrical engineering from the Republic of Korea Airforce in 1981. He received his Master's degree and Ph.D. in electrical engineering from Florida Institute of Technology in 1987 and 1993, respectively. He worked in the areas of information and communications in the Republic of Korea Airforce from 1981 to 2004. Since 2005, he has worked for the ISR systems department at LIG Nex1. His current research interests include radar signal processing, target tracking, and system engineering.

Chulhun Seo (M'97-SM'14)

Please refer to the July 2017 issue (JEES vol. 16, no. 3).
[14] M. I. Skolnik, Radar Handbook, 2nd ed. Singapore: McGraw-Hill, 1991.
Jeongho Park

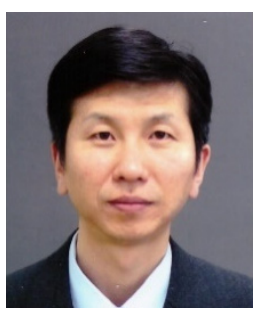

received his B.S. degree in electrical engineering from Yonsei University in 1988 and his Master's degree and Ph.D. in electrical engineering from POSTECH in 1990 and 2001, respectively. Since 1990, he has worked in the development of advanced radar systems at LIG Nex1. His current research interests include radar signal processing, target tracking, and system engineering.
Dong-Hui Lee

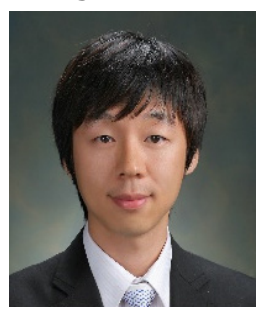

received B.S. and M.S. degrees in telecommunication engineering from Korea Aerospace University in 2009 and 2011, respectively. Since 2011, he has worked in the development of advanced radar systems at LIG Nex1. His current research interests include radar signal processing, tracking filters, and system engineering. 
NGUYEN et al.: ANALYSIS OF THE OPTIMAL FREQUENCY BAND FOR A BALLISTIC MISSILE DEFENSE RADAR SYSTEM

\section{APPENDIX}

Calculate Jacobian matrix $\mathbf{G}$, which is in the following form:

$$
\mathbf{G}=\left[\begin{array}{lllllll}
G_{11} & G_{12} & G_{13} & 0 & 0 & 0 & 0 \\
G_{21} & G_{22} & G_{23} & 0 & 0 & 0 & 0 \\
G_{31} & G_{32} & G_{33} & 0 & 0 & 0 & 0
\end{array}\right]
$$

$G_{11}=\frac{1}{r}\left(x_{1}^{\prime} \cos \lambda+x_{2}^{\prime} \cos \phi \sin \lambda+x_{3}^{\prime} \sin \phi \sin \lambda\right)$

$G_{12}=\frac{1}{r}\left(-x_{1}^{\prime} \sin \lambda+x_{2}^{\prime} \cos \phi \cos \lambda+x_{3}^{\prime} \sin \phi \cos \lambda\right)$

$G_{13}=\frac{1}{r}\left(-x_{2}^{\prime} \sin \phi+x_{3}^{\prime} \cos \phi\right)$

$G_{21}=\frac{1}{x_{1}^{\prime 2}+x_{2}^{\prime 2}}\left(x_{1}^{\prime} \cos \phi \sin \lambda-x_{2}^{\prime} \cos \lambda\right)$

$G_{22}=\frac{1}{x_{1}^{\prime 2}+x_{2}^{\prime 2}}\left(x_{1}^{\prime} \cos \phi \cos \lambda+x_{2}^{\prime} \sin \lambda\right)$

$G_{23}=\frac{1}{x_{1}^{\prime 2}+x_{2}^{\prime 2}}\left(-x_{1}^{\prime} \sin \phi\right)$

$$
G_{31}=\frac{1}{r^{2}}\left(\sqrt{x_{1}^{\prime 2}+x_{2}^{\prime 2}} \sin \phi \sin \lambda-x_{3}^{\prime}\left(x_{1}^{\prime} \cos \lambda\right.\right.
$$$$
\left.\left.+x_{2}^{\prime} \cos \phi \sin \lambda\right) / \sqrt{x_{1}^{\prime 2}+x_{2}^{\prime 2}}\right)
$$$$
G_{32}=\frac{1}{r^{2}}\left(\sqrt{x_{1}^{\prime 2}+x_{2}^{\prime 2}} \sin \phi \cos \lambda-x_{3}^{\prime}\left(x_{2}^{\prime} \cos \phi \cos \lambda-\right.\right.
$$
$\left.\left.x_{1}^{\prime} \sin \lambda\right) / \sqrt{x_{1}^{\prime 2}+x_{2}^{\prime 2}}\right)$

$$
\begin{aligned}
G_{33}= & \frac{1}{r^{2}}\left(\sqrt{x_{1}^{\prime 2}+x_{2}^{\prime 2}} \cos \phi-x_{1}^{\prime}\left(x_{2}^{\prime 2} \sin \phi\right) /\right. \\
& \left.\sqrt{x_{1}^{\prime 2}+x_{2}^{\prime 2}}\right)
\end{aligned}
$$

where

$$
\begin{gathered}
{\left[\begin{array}{l}
x_{1}^{\prime} \\
x_{2}^{\prime} \\
x_{3}^{\prime}
\end{array}\right]=\mathbf{T}^{-1}\left[\begin{array}{l}
x_{1} \\
x_{2} \\
x_{3}
\end{array}\right]} \\
r=\sqrt{x_{1}^{\prime 2}+x_{2}^{\prime 2}+x_{3}^{\prime 2}}
\end{gathered}
$$

